\title{
Ketone functionalized task specific ionic liquids for selective tantalum Extraction
}

\author{
R. Turgis ${ }^{a}$, G. Arracharta ${ }^{a, *}$, S. Michelb, S. Legeai ${ }^{b}$, M. Lejeune ${ }^{c}$, M. Draye ${ }^{c}$, S. Pellet-Rostainga ${ }^{a, *}$ \\ a ICSM-UMR 5257, CEA, CNRS, Univ. Montpellier, ENSCM, Bagnols sur Cèze, France \\ ${ }^{\mathrm{b}}$ IJL-UMR CNRS 7198, Univ. Lorraine, Groupe Chimie et Electrochimie des Matériaux, Metz, France \\ ' LCME, Univ. Savoie Mont Blanc, Le Bourget du Lac, France \\ *Corresponding author. E-mail address: guilhem.arrachart@cea.fr , stephane.pellet-rostaing@cea.fr
}

\begin{abstract}
A novel family of ketone functionalized task specific ionic liquids (TSILs) was designed for the selective extraction of tantalum from sulfuric acid medium. We report the synthesis of functionalized hydrophobic ionic liquids based on piperidium, imidazolium or pyridinium cations bearing ketone moieties and their application in the liquid/liquid extraction of $\mathrm{Ta}(\mathrm{V})$. Recovery processes for Ta after extraction were performed and the recyclability of the TSIL was demonstrated. An eco-friendly process based on a TSIL was proposed for the extraction and stripping of tantalum from sulphuric acidic medium. Such system can be used as an alternative to the methyl-iso-butyl ketone (MIBK) which is the most commonly used extractant for the separation and purification of niobium and tantalum by solvent extraction.
\end{abstract}

\section{Keywords}

Task specific ionic liquids; Tantalum; Selective ionic separation

\section{Introduction}

Tantalum ( $\mathrm{Ta}$ ) and niobium ( $\mathrm{Nb}$ ) are transition metals with very similar physical and chemicals properties and almost always associated geologically [1]. These rare refractory metals are important and widely used in steel, electronic and other high-tech industries [2]. Tantalum is mainly used in electronic industry where about $60 \%$ of the annual tantalum production is consumed principally to produce powders for capacitors. Due to technological needs, the Ta demand continues to increase year by year in order to supply the capacitors and semiconductor for electronics devices production. These applications and many others such as surgical implants, cemented carbides and superalloys, make Ta economically and strategically important to industrialised countries (European Commission, 2011, 2014). New sources of supply may be developed for strategic reasons such as diversify geographic location of supply or efforts to recycle this element from manufacturing scrap [3]. Also an interesting way which is not yet explore is to recycle it from electronic capacitors particularly from end of life products such as the Waste of Electrical and Electronic Equipments (WEEE).

$\mathrm{Ta}$ and $\mathrm{Nb}$ are difficult to separate because of their shared physical and chemical properties. Liquidliquid extraction is currently the most effective way of separating tantalum and niobium. Usually, niobium - tantalum concentrate is digested using a mixture of hydrofluoric and sulphuric acids [4], and then can be extracted and separated from such acidic leach liquors by using organic solvents such as methyl iso-butyl ketone (MIBK), tri-butyl phosphate (TBP), cyclohexanone and 2-Octanol [5]. MIBK is the most commonly used extractant due to its high selectivity for tantalum and niobium, its low density and low viscosity. However, MIBK is fire- and explosion-hazardous and readily soluble in aqueous solutions, which are major disadvantages for such process. 
Room-temperature ionic liquids (ILS), which are usually considered as nonflammable, nonvolatile as well are exhibiting good thermal stability and are able to solubilize a wide range of solutes; they can be used as alternative diluents or extractants of volatile organic compounds in the extraction process [6-8]. Since Dai et al. [9] work showing the potential of ILs for liquid-liquid extraction, many studies have been developed using various extractants or synergistic systems diluted in ILs or using "task specific ionic liquids" (TSILS) [10-14]. The TSILs, which consist of extracting moieties grafted onto the cation of the ionic liquid, combine the properties of ionic liquids with those of conventional extracting compounds. Moreover TSILs can act both as the organic phase as well as the extracting agent, avoiding the difficulties that can be encountered through extractant/solvent miscibility.

In most of the examples described in the literature, the IL-based extraction systems are more efficient and evaluated as preferable than traditional organic solvent [15-17]. Usually it appears also that the extraction mechanism is very different $[15,18]$. Depending on the nature of the ligand, the IL and the aqueous phase, mechanisms such as ion-exchange, neutral coextraction or neutral solvation can be encountered [19]. To our knowledge there is no system describing the separation and purification of Ta by liquid liquid extraction using ionic liquids. Only few examples of ILs systems have been describe for the electrodeposition of Ta $[20,21]$.

Considering the limitations associated to the physico-chemicals properties of MIBK and the potential of ILs for liquid-liquid extraction, we chose to design and synthesize task specific ionic liquid TSIL for the competitive extraction and purification of Ta from sulphuric acid solution, in the presence of elements such as $\mathrm{Nb}$ and elements that may be present in acidic leach liquors of capacitors. Various functionalized hydrophobic ionic liquids based on piperidium, imidazolium or pyridinium cations bearing ketone moieties have been synthesized for the liquid/liquid extraction of $\mathrm{Ta}(\mathrm{V})$.

\section{Experimental}

\subsection{Materials and reagents}

All the chemicals and reagents used in the study were of analytical grade and purchased from SigmaAldrich or Alfa Aesar. They were used without further purification. Anhydrous solvents were purchased from Acros (AcroSeal ${ }^{\circledR}$ ). ICP calibration and verification standards were purchased from SCP Sciences.

The density measurements were performed with a DSA 5000 thermo-regulated digital densimeter (Anton Paar). The apparatus measures densities using an oscillating U-tube with an accuracy of 0.00001 $\mathrm{g} / \mathrm{cm} 3$ and temperature controlled to within $0.001^{\circ} \mathrm{C}$. All measurements were conducted at $25.005 \pm$ $0.004^{\circ} \mathrm{C}$.

For the viscosity measurements, an Anton Paar MCR 301 rheometer was used. A plate-plate geometry (PP25, diameter 24.981) was chosen to measure shear viscosities under thermostatic control $\left(25^{\circ} \mathrm{C}\right)$ from shear rates of 0.1 to $300 \mathrm{l} / \mathrm{s}$. Shear rates were increased with 10 points per decade. The measurement duration per point was set to $6 \mathrm{~s}$.

Acid concentration in the aqueous and TSIL phases before and after extraction was analysed using a Metrohm 809 Titrando by titration with $\mathrm{NaOH} 0.1$ or $0.01 \mathrm{M}$ (Fluka analytical).

The solubility of the TSIL in the aqueous phase was determined by measuring the concentrations of the cation in the aqueous solutions by measuring the Total Organic Content (TOC) using a Shimadzu TOCVCSH analyser based on a $680^{\circ} \mathrm{C}$ combustion catalytic oxidation/NDIR method.

The solubility of water in the TSILs was determined by Karl Fischer coulometric titration using a

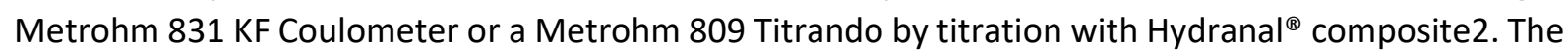


TSIL was contacted with the aqueous phase for $1 \mathrm{~h}$ and the two-phase system was then centrifuged and the TSIL water content was measured. NMR analyses were performed on a Bruker 400 ultrashield VS spectrometer. Displacements are reported in ppm using the solvent $(\mathrm{CDCl} 3: 7.26 \mathrm{ppm} 1 \mathrm{H} ; 77.16$ ppm 13C) as an internal reference. The purity of the various compounds have been determined thanks to the NMR analysis and be assumed to be $\geq 95 \%$.

Metal concentrations were measured by inductively coupled plasma/atomic emission spectroscopy (ICP/AES) using a SPECTRO ARCOS spectrometer.

\subsection{Synthesis of TSILS}

\subsubsection{General procedure for $\alpha$-bromoketone (compounds A)}

A mixture of $100 \mathrm{mmol}$ of ketone $\mathrm{CH}_{3} \mathrm{C}(\mathrm{O}) \mathrm{R}^{1}$ with $10 \mathrm{mmol}$ of $\mathrm{p}$-Toluenesulfonic acid ( $\mathrm{p}-\mathrm{TsOH}$ ) in 100 $\mathrm{mL}$ of ethyl acetate was stirred under nitrogen atmosphere. $100 \mathrm{mmol}$ of $\mathrm{N}$-Bromosuccinimide were then added to the reaction mixture, that was stirred for $4 \mathrm{~h}$ at room temperature and protected from light. The mixture was then filtered, the organic phase was washed successively with a $1 \mathrm{M}$ potassium carbonate solution, a saturated sodium thiosulfate solution and with water ( 2 times). The organic phase was then dried over sodium sulfate and concentrated under vacuum. The $\alpha$-brominated ketones were obtained as yellow oil.

\subsubsection{A1: Br-MIBK, 1-bromo-4-methyl-pent-2-one (yield 61\%).}

$\mathrm{Br}-\mathrm{MIBK}$ was prepared starting from methyl iso-butyl ketone (MIBK), and was then obtained in admixture with 3-bromo-4-methyl-pentan-2-one in a 65/35 ratio. Caution/extremely tear-inducing.

${ }^{1} \mathrm{H} \mathrm{NMR}(400 \mathrm{MHz}, \mathrm{CDCl}) \delta(\mathrm{ppm}): 0.92(\mathrm{~d}, 6 \mathrm{H}, \mathrm{J}=6.7 \mathrm{~Hz}, \mathrm{CH} 3) ; 2.18(\mathrm{~m}, 1 \mathrm{H}, \mathrm{CH}) ; 2.51(\mathrm{~d}, 2 \mathrm{H}, \mathrm{J}=7.0$ $\mathrm{Hz}, \mathrm{C}(\mathrm{O})-\mathrm{CH} 2) ; 3.86$ (s, $2 \mathrm{H}, \mathrm{Br}-\mathrm{CH} 2-\mathrm{C}(\mathrm{O}))$

\subsubsection{A2: Br-AcPh, 2-bromo-acetophenone (yield 95\%).}

$\mathrm{Br}-\mathrm{AcPh}$ was prepared starting from acetophenone (AcPh).

${ }^{1} \mathrm{H}$ NMR (400 MHz, CDCl3) $\delta(\mathrm{ppm}): 4.56$ (s, $\left.2 \mathrm{H}, \mathrm{Br}-\mathrm{CH} 2-\mathrm{C}(\mathrm{O})\right) ; 7.49$ (t, $\left.2 \mathrm{H}, \mathrm{J}=7.5 \mathrm{~Hz}, \mathrm{CHAr}\right) ; 7.61$ (t, 2 $\mathrm{H}, \mathrm{J}=7.5 \mathrm{~Hz}, \mathrm{CHAr}) ; 7.98(\mathrm{~d}, 2 \mathrm{H}, \mathrm{J}=7.5 \mathrm{~Hz}, \mathrm{CHAr}$ ).

\subsubsection{A3: Br-MacPh, 2-bromo-4'-methyl-acetophenone (yield 95\%).}

$\mathrm{Br}-\mathrm{MacPh}$ was then prepared starting from 4-methyl-acetophenone (MAcPh).

${ }^{1} \mathrm{H} \mathrm{NMR}(400 \mathrm{MHz}, \mathrm{CDCl} 3) \delta(\mathrm{ppm}): 2.42(\mathrm{~s}, 3 \mathrm{H}, \mathrm{CH} 3) ; 4.43(\mathrm{~s}, 2 \mathrm{H}, \mathrm{Br}-\mathrm{CH} 2-\mathrm{C}(\mathrm{O})) ; 7.29$ (d, $2 \mathrm{H}, \mathrm{J}=8.0 \mathrm{~Hz}$, CHAr); 7.88 (d, $2 \mathrm{H}, \mathrm{J}=8.0 \mathrm{~Hz}, \mathrm{CHAr})$.

\subsubsection{Synthesis of pentan-2-one derivatives}

\subsubsection{5-bromopentan-2-one (yield 80\%).}

To a solution of $10 \mathrm{~g}$ of acetylbutyrolactone $(78 \mathrm{mmol})$ in toluene were added $13.5 \mathrm{~mL}$ of $48 \%$ hydrobromic acid $(117 \mathrm{mmol})$. The mixture was heated to $80^{\circ} \mathrm{C}$ overnight. After cooling, the biphasic mixture was separated and the aqueous phase was extracted twice with diethyl ether. The organic phases were then combined, washed twice with water, dried over magnesium sulfate and concentrated. After distillation under reduced pressure $\left(100^{\circ} \mathrm{C}\right.$. under $20 \mathrm{~mm}$ bar), 5-bromopentan 2one (12.7 g) was obtained as a colorless oil.

${ }^{1} \mathrm{H}$ NMR (400 MHz, CDCl3) $\delta$ (ppm): 2.11 (m, $\left.2 \mathrm{H},(\mathrm{O}) \mathrm{C}-\mathrm{CH} 2-\mathrm{CH} 2-\mathrm{CH} 2-\mathrm{Br}\right) ; 2.16$ (s, $\left.3 \mathrm{H}, \mathrm{CH} 3-(\mathrm{O}) \mathrm{C}\right) ; 2.63$ (t, J = 7.0 Hz, $2 \mathrm{H},(\mathrm{O}) \mathrm{CCH} 2-\mathrm{CH} 2-\mathrm{CH} 2-\mathrm{Br}) ; 3.44(\mathrm{t}, \mathrm{J}=6.5 \mathrm{~Hz}, 2 \mathrm{H},(\mathrm{O}) \mathrm{C}-\mathrm{CH} 2-\mathrm{CH} 2-\mathrm{CH} 2-\mathrm{Br}$ ).

\subsubsection{5-(Piperidinyl)pentan-2-one (yield 97\%).}

To a solution of $2.4 \mathrm{~mL}$ of piperidine $(23.7 \mathrm{mmol})$ in $30 \mathrm{~mL}$ of dry acetonitrile under an inert atmosphere, were successively added potassium carbonate $(6.0 \mathrm{~g}, 43 \mathrm{mmol})$ and a solution of 5bromopentan-2-one $(3.56 \mathrm{~g}, 21.6 \mathrm{mmol})$ in $10 \mathrm{~mL}$ of dry acetonitrile. The mixture was then heated at 
$80^{\circ} \mathrm{C}$ for $2 \mathrm{~h}$. After cooling, the mixture was filtered and the salts were washed with acetonitrile. The combined organic filtrate was then concentrated under vacuum leading 5-(Piperidinyl) pentan-2- one $(3.53 \mathrm{~g})$ as a slightly yellow oil.

${ }^{1} \mathrm{H}$ NMR $(400 \mathrm{MHz}, \mathrm{CDCl} 3) \delta$ (ppm): 1.39 (m, $\left.2 \mathrm{H}, \mathrm{CH} 2-\mathrm{CH} 2-\mathrm{CH} 2-\mathrm{N}\right) ; 1.53(\mathrm{~m}, 4 \mathrm{H}, \mathrm{CH} 2-\mathrm{CH} 2-\mathrm{CH} 2-\mathrm{N}) ; 1.74$ (m, $2 \mathrm{H},(\mathrm{O}) \mathrm{C}-\mathrm{CH} 2-\mathrm{CH} 2-\mathrm{CH} 2-\mathrm{N}) ; 2.13(\mathrm{~s}, 3 \mathrm{H}, \mathrm{CH} 3-(\mathrm{O}) \mathrm{C}) ; 2.24$ (t, J = $7.5 \mathrm{~Hz}, 2 \mathrm{H},(\mathrm{O}) \mathrm{C}-\mathrm{CH} 2-\mathrm{CH} 2-\mathrm{CH} 2-\mathrm{N})$; 2.32 (bs, $4 \mathrm{H}, \mathrm{CH} 2-\mathrm{CH} 2-\mathrm{CH} 2-\mathrm{N}) ; 2.41$ (t, J = $7.0 \mathrm{~Hz}, 2 \mathrm{H},(\mathrm{O}) \mathrm{C}-\mathrm{CH} 2-\mathrm{CH} 2-\mathrm{CH} 2-\mathrm{N})$.

${ }^{13} \mathrm{C} \mathrm{NMR}(100 \mathrm{MHz}, \mathrm{CDCl} 3) \delta(\mathrm{ppm}): 21.3((\mathrm{O}) \mathrm{C}-\mathrm{CH} 2-\mathrm{CH} 2-\mathrm{CH} 2-\mathrm{N}) ; 24.5(\mathrm{CH} 2-\mathrm{CH} 2-\mathrm{CH} 2-\mathrm{N}) ; 26.0(\mathrm{CH} 2$ $\mathrm{CH} 2-\mathrm{CH} 2-\mathrm{N}) ; 30.1(\mathrm{C}(\mathrm{O})-\mathrm{CH} 3) ; 41.8((\mathrm{O}) \mathrm{C}-\mathrm{CH} 2-\mathrm{CH} 2-\mathrm{CH} 2-\mathrm{N}) ; 54.6(\mathrm{CH} 2-\mathrm{CH} 2-\mathrm{CH} 2-\mathrm{N}) ; 58.6$ ((O)C-CH2-

$\mathrm{CH} 2-\mathrm{CH} 2-\mathrm{N}) ; 209.0(\mathrm{C}(\mathrm{O}))$.

\subsubsection{General procedure for the alkylation of haloketone (compound $B$ )}

To a mixture of $105 \mathrm{mmol}$ of $\alpha$-haloketone in $100 \mathrm{~mL}$ of ethyl acetate was added dropwise the desired amine $(126 \mathrm{mmol})$ under argon at room temperature, over $30 \mathrm{~min}$ under vigorous stirring. The reaction mixture was then stirred at room temperature for $12 \mathrm{~h}$. After filtration, the salts were washed with ethyl acetate and the combined organic filtrate was then concentrated under vacuum. The desired compounds were obtained as white powder.

2.2.3.1. B1a: [EPip-MIBK]Br, N-ethyl-N-(4-methyl-2-oxopentyl)piperidinium bromide (yield 75\%). [EPipMIBK]Br was prepared starting from compound A1 (31.5 g of mixture of 1-bromo-4-methyl-pentan-2one and 3-bromo-4-methyl-pentan-2-one corresponding to $18.87 \mathrm{~g}$ of 1-bromo-4-methyl-pentan-2one) and 1-ethylpiperidine (17 mL).

${ }^{1} \mathrm{H}$ NMR $(400 \mathrm{MHz}, \mathrm{CDCl} 3) \delta(\mathrm{ppm}): 0.76(\mathrm{~d}, 6 \mathrm{H}, \mathrm{J}=6.5 \mathrm{~Hz}, \mathrm{CH} 3) ; 1.18(\mathrm{t}, 3 \mathrm{H}, \mathrm{J}=7.5 \mathrm{~Hz}, \mathrm{CH} 3-\mathrm{CH} 2-\mathrm{N})$; 1.61-1.86 (m, $6 \mathrm{H}, \mathrm{CH} 2) ; 2.01(\mathrm{~m}, 1 \mathrm{H}, \mathrm{CH}) ; 2.39(\mathrm{~d}, 2 \mathrm{H}, \mathrm{J}=7.0 \mathrm{~Hz}, \mathrm{C}(\mathrm{O})-\mathrm{CH} 2) ; 3.54(\mathrm{~m}, 2 \mathrm{H}, \mathrm{CH} 2-\mathrm{N}) ; 3.71$ (q, $2 \mathrm{H}, \mathrm{J}=7.5 \mathrm{~Hz}, \mathrm{CH} 3-\mathrm{CH} 2-\mathrm{N}) ; 3.88(\mathrm{~m}, 2 \mathrm{H}, \mathrm{CH} 2-\mathrm{N})$; 4.86 (s, $2 \mathrm{H}, \mathrm{N}-\mathrm{CH} 2-\mathrm{C}(\mathrm{O}))$.

${ }^{13} \mathrm{C} \mathrm{NMR}(100 \mathrm{MHz}, \mathrm{CDCl} 3) \delta$ (ppm): 8.2 (CH3); 19.7; $20.6(\mathrm{CH} 2) ; 22.3(\mathrm{CH} 3) ; 24.0(\mathrm{CH}) ; 49.9$ (C(O) -CH2); $53.8(\mathrm{CH} 3-\mathrm{CH} 2-\mathrm{N}) ; 59.2(\mathrm{CH} 2-\mathrm{N}) ; 63.1(\mathrm{~N}-\mathrm{CH} 2-\mathrm{C}(\mathrm{O})) ; 202.3(\mathrm{C}(\mathrm{O}))$.

2.2.3.2. B1b: [MIm-MIBK]Br, N-methyl-N-(4-methyl-2-oxopentyl)imidazolium bromide (yield $80 \%$ ). [MIm-MIBK]Br was prepared starting from compound A1 (31.5 g of mixture of 1-bromo-4-methylpentan-2- one and 3-bromo-4-methyl-pentan-2-one corresponding to $18.87 \mathrm{~g}$ of 1-bromo-4-methylpentan-2-one) and 1-methylimidazole (10 $\mathrm{mL})$.

${ }^{1} \mathrm{H} \mathrm{NMR} \mathrm{(400} \mathrm{MHz,} \mathrm{CDCl3)} \delta(\mathrm{ppm}): 0.91(\mathrm{~d}, 6 \mathrm{H}, \mathrm{J}=6.5 \mathrm{~Hz}, \mathrm{CH} 3) ; 2.15(\mathrm{~m}, 1 \mathrm{H}, \mathrm{CH}) ; 2.53(\mathrm{~d}, 2 \mathrm{H}, \mathrm{J}=7.0$ $\mathrm{Hz}, \mathrm{C}(\mathrm{O})-\mathrm{CH} 2) ; 4.02(\mathrm{~s}, 3 \mathrm{H}, \mathrm{CH} 3-\mathrm{N}) ; 5.67(\mathrm{~s}, 2 \mathrm{H}, \mathrm{N}-\mathrm{CH} 2-\mathrm{C}(\mathrm{O})$ ); 7.47 (q, $1 \mathrm{H}, \mathrm{J}=2.0 \mathrm{~Hz}, \mathrm{CHAr}$ ); 7.61 (q, $1 \mathrm{H}, \mathrm{J}=2.0 \mathrm{~Hz}, \mathrm{CHAr}) ; 9.78(\mathrm{~s}, 1 \mathrm{H}, \mathrm{J}=2.0 \mathrm{~Hz}, \mathrm{CHAr}$ ).

${ }^{13} \mathrm{C} \mathrm{NMR}(100 \mathrm{MHz}, \mathrm{CDCl} 3) \delta$ (ppm): 22.6 (CH3); 24.2 (CH); 36.9 (CH3-Ar); 48.9 (C(O)-CH2); 60.0 (N-CH2C(O)); 122.7 (CHAr); 124.1 (CHAr); 137.9 (CHAr); 201.7 (C(O)).

\subsubsection{B1c: [Py-MIBK]Br, N-(4-methyl-2-oxopentyl)pyridinium bromide (yield 80\%).}

[Py-MIBK]Br was prepared starting from compound A1 (31.5 g of mixture of 1-bromo-4-methylpentan-2-one and 3-bromo-4- methyl-pentan-2-one corresponding to $18.87 \mathrm{~g}$ of 1-bromo-4methylpentan-2-one) and pyridine $(10.2 \mathrm{~mL})$.

${ }^{1} \mathrm{H} \mathrm{NMR}(400 \mathrm{MHz}, \mathrm{CDCl} 3) \delta(\mathrm{ppm}): 0.91(\mathrm{~d}, 6 \mathrm{H}, \mathrm{J}=6.5 \mathrm{~Hz}, \mathrm{CH} 3) ; 2.19(\mathrm{~m}, 1 \mathrm{H}, \mathrm{CH}) ; 2.57(\mathrm{~d}, 2 \mathrm{H}, \mathrm{J}=7.0$ $\mathrm{Hz}, \mathrm{C}(\mathrm{O})-\mathrm{CH} 2) ; 6.51(\mathrm{~s}, 2 \mathrm{H}, \mathrm{NCH} 2-\mathrm{C}(\mathrm{O})) ; 8.04(\mathrm{t}, 2 \mathrm{H}, \mathrm{J}=7.0 \mathrm{~Hz}, \mathrm{CHAr}) ; 8.49$ (q, $1 \mathrm{H}, \mathrm{J}=7.0 \mathrm{~Hz}$,

CHAr); $9.31(\mathrm{~d}, 2 \mathrm{H}, \mathrm{J}=6.0 \mathrm{~Hz}, \mathrm{CHAr})$.

${ }^{13} \mathrm{C}$ NMR (100 MHz, CDCl3) $\delta$ (ppm): 22.7 (CH3); $24.1(\mathrm{CH}) ; 49.0(\mathrm{C}(\mathrm{O})-\mathrm{CH} 2) ; 66.6(\mathrm{~N}-\mathrm{CH} 2-\mathrm{C}(\mathrm{O})) ; 127.6$ (CHAr); 145.5 (CHAr); 146.3 (CHAr); 200.3 (C(O)).

\subsubsection{B2: [EPip-AcPh]Br, N-ethyl-N-acetophenone piperidinium bromide (yield 95\%).}

[EPip-AcPh]Br was prepared starting from compound $\mathrm{A} 2(20.8 \mathrm{~g})$ and 1-ethylpiperidine $(17 \mathrm{~mL})$. 1H NMR (400 MHz, CDCl3) $\delta$ (ppm): 1.31 (t, $3 \mathrm{H}, \mathrm{J}=7.5 \mathrm{~Hz}, \mathrm{CH} 3-\mathrm{CH} 2-\mathrm{N}) ; 1.79(\mathrm{~m}, 2 \mathrm{H}, \mathrm{CH}$ ); 1.91 (m, 4 $\mathrm{H}, \mathrm{CH} 2) ; 3.83(\mathrm{dt}, 2 \mathrm{H}, \mathrm{J}=12.5 \mathrm{~Hz}, \mathrm{~J}=4.5 \mathrm{~Hz}, \mathrm{~m}, \mathrm{CH} 2-\mathrm{CH} 2-\mathrm{N}) ; 3.94(\mathrm{~m}, 2 \mathrm{H}, \mathrm{J}=7.5 \mathrm{~Hz}$, 
$\mathrm{CH} 3-\mathrm{CH} 2-\mathrm{N}) ; 4.38(\mathrm{~m}, 2 \mathrm{H}, \mathrm{CH} 2-\mathrm{N}) ; 5.62(\mathrm{~s}, 2 \mathrm{H}, \mathrm{N}-\mathrm{CH} 2-\mathrm{C}(\mathrm{O})) ; 7.44(\mathrm{t}, 2 \mathrm{H}, \mathrm{J}=7.5 \mathrm{~Hz}, \mathrm{CHAr}) ; 7.57$ (t, $1 \mathrm{H}$, $\mathrm{J}=7.5 \mathrm{~Hz}, \mathrm{CHAr}) ; 8.19$ (d, $2 \mathrm{H}, \mathrm{J}=7.5 \mathrm{~Hz}, \mathrm{CHAr})$.

${ }^{13} \mathrm{C} \mathrm{NMR}(100 \mathrm{MHz}, \mathrm{CDCl} 3) \delta(\mathrm{ppm}): 8.4(\mathrm{CH} 3) ; 20.1 ; 20.7(\mathrm{CH} 2) ; 53.2(\mathrm{CH} 3-\mathrm{CH} 2-\mathrm{N}) ; 59.7$ (CH2-N); 61.6 (N-CH2-C(O)); 128.8; 129.1 (CHAr); 134.1 (CAr); 135.0 (CHAr); 191.5 (C(O)).

2.2.3.5. B3: [Epip-MAcPh]Br, N-ethyl-N-4'-methylacetophenone piperidinium bromide (yield 88\%). [Epip-MAcPh]Br was prepared starting from compound $\mathrm{A} 3(22.3 \mathrm{~g})$ and 1-ethylpiperidine (17 $\mathrm{mL})$.

${ }^{1} \mathrm{H}$ NMR $(400 \mathrm{MHz}, \mathrm{CDCl} 3) \delta(\mathrm{ppm}): 1.30(\mathrm{t}, 3 \mathrm{H}, \mathrm{J}=7.5 \mathrm{~Hz}, \mathrm{CH} 3-\mathrm{CH} 2-\mathrm{N}) ; 1.79-1.91(\mathrm{~m}, 6 \mathrm{H}, \mathrm{CH} 2) ; 2.34$ (s, $3 \mathrm{H}, \mathrm{CH} 3-\mathrm{Ar}) ; 3.81(\mathrm{dt}, 2 \mathrm{H}, \mathrm{J}=12.5 \mathrm{~Hz}, \mathrm{~J}=4.5 \mathrm{~Hz}, \mathrm{~m}, \mathrm{CH} 2-\mathrm{CH} 2-\mathrm{N}) ; 3.93(\mathrm{~m}, 2 \mathrm{H}, \mathrm{J}=7.5 \mathrm{~Hz}, \mathrm{CH} 3-\mathrm{CH} 2-$ $\mathrm{N}) ; 4.38(\mathrm{~m}, 2 \mathrm{H}, \mathrm{CH} 2-\mathrm{N}) ; 5.55(\mathrm{~s}, 2 \mathrm{H}, \mathrm{N}-\mathrm{CH} 2-\mathrm{C}(\mathrm{O})) ; 7.23(\mathrm{~d}, 2 \mathrm{H}, \mathrm{J}=8.0 \mathrm{~Hz}, \mathrm{CHAr}) ; 8.07(\mathrm{~d}, 2 \mathrm{H}, \mathrm{J}=8.0$ $\mathrm{Hz}, \mathrm{CHAr})$.

${ }^{13} \mathrm{C} \mathrm{NMR}$ (100 MHz, CDCl3) $\delta$ (ppm): 8.4 (CH3); 20.1; 20.7 (CH2); 21.8 (CH3-Ar); 53.2 (CH3-CH2-N); 59.7 (CH2-N); 61.4 (N-CH2-C(O)); 128.9; 129.8 (CHAr); 131.6 (CAr); 146.2 (CHAr); 190.9 (C(O)).

2.2.3.6. B4: [EPip-Pin]l, N-ethyl-N-pinacolone piperidinium iodide (yield 80\%).

[EPip-Pin]l was prepared starting from 1-iodopinacolone $(4.5 \mathrm{~g}, 20 \mathrm{mmol})$ and 1-ethylpiperidine (3.3 $\mathrm{mL}, 24 \mathrm{mmol})$. The mixture is heated at $50^{\circ} \mathrm{C}$ for $12 \mathrm{~h}$.

${ }^{1} \mathrm{H}$ NMR $(400 \mathrm{MHz}, \mathrm{CDCl} 3) \delta(\mathrm{ppm}): 1.26(\mathrm{~s}, 9 \mathrm{H}, \mathrm{CH} 3) ; 1.31(\mathrm{t}, 3 \mathrm{H}, \mathrm{J}=7.5 \mathrm{~Hz}, \mathrm{CH} 3-\mathrm{CH} 2-\mathrm{N}) ; 1.74-1.98$ (m, $6 \mathrm{H}, \mathrm{CH} 2) ; 3.73-3.84(\mathrm{~m}, 4 \mathrm{H}, \mathrm{CH} 2-\mathrm{N}$; $\mathrm{CH} 3-\mathrm{CH} 2-\mathrm{N}) ; 4.20(\mathrm{~m}, 2 \mathrm{H}, \mathrm{CH} 2-\mathrm{N}) ; 5.07(\mathrm{~s}, 2 \mathrm{H}, \mathrm{N}-\mathrm{CH} 2-\mathrm{C}(\mathrm{O}))$. ${ }^{13} \mathrm{C} \mathrm{NMR}(100 \mathrm{MHz}, \mathrm{CDCl} 3) \delta(\mathrm{ppm}): 8.6(\mathrm{CH} 3-\mathrm{CH} 2-\mathrm{N}) ; 20.0 ; 20.5(\mathrm{CH} 2) ; 26.6(\mathrm{CH} 3) ; 45.0(\mathrm{C}(\mathrm{O})-\mathrm{C}(\mathrm{CH} 3) 3)$; 52.5 (CH3-CH2-N); 59.6 (CH2-N; N-CH2-C(O)); 208.1 (C(O)).

\subsubsection{B5: [MIm-Pin]Cl, N-pinacolone methylimidazolium chloride (yield 80\%).}

[MIm-Pin]Cl was prepared starting from 1-chloropinacolone $(2.7 \mathrm{~g}, 20 \mathrm{mmol})$ and 1-methylimidazole (1.9 mL, $24 \mathrm{mmol})$.

${ }^{1} \mathrm{H} \mathrm{NMR} \mathrm{(400} \mathrm{MHz,} \mathrm{CDCl3)} \delta$ (ppm): 1.20 (s, $9 \mathrm{H}, \mathrm{CH} 3$ ); 3.96 (s, $\left.3 \mathrm{H}, \mathrm{CH} 3-\mathrm{Ar}\right) ; 5.84$ (s, $2 \mathrm{H}, \mathrm{N}-\mathrm{CH} 2-\mathrm{C}(\mathrm{O})$ ); 7.49 (s, $1 \mathrm{H}, \mathrm{CHAr}) ; 7.62$ (s, $1 \mathrm{H}, \mathrm{CHAr}) ; 10.07$ (s, $1 \mathrm{H}, \mathrm{CHAr}$ ).

${ }^{13} \mathrm{C} \mathrm{NMR}(100 \mathrm{MHz}, \mathrm{CDCl} 3) \delta$ (ppm): 26.2 (CH3); 36.6 (CH3-N); 43.4 ((CH3)3-C-C(O)); 54.3 (C(O)-CH2); 122.5 (CHAr); 124.2 (CHAr); 138.4 (CHAr); 206.8 (C(O)).

\subsubsection{B6: [EPipP4K]Br, 1-ethyl-4-oxopentyl-piperidinium bromide (yield 95\%).}

To a solution of 5-(piperidinyl) pentan-2-one $(3.53 \mathrm{~g}, 20.1 \mathrm{mmol})$ in $10 \mathrm{~mL}$ of acetonitrile under an inert atmosphere was added bromoethane $(3.3 \mathrm{~mL}, 40 \mathrm{mmol})$. The mixture was then heated at $80^{\circ} \mathrm{C}$ for 12 h. After cooling, the mixture was concentrated under vacuum. The resulting solid was dissolved in dichloromethane and reprecipitated by addition of ethyl acetate. The solid was then washed twice with ethyl acetate and dried under vacuum. [EPIPP4 K] Br ( $5.28 \mathrm{~g})$ was then obtained as a slightly yellow solid.

${ }^{1} \mathrm{H}$ NMR (400 MHz, CDCl3) $\delta$ (ppm): 1.35 (t, J =7.5 Hz, CH3-CH2-N); 1.74 (m, $\left.2 \mathrm{H}, \mathrm{CH} 2-\mathrm{CH} 2-\mathrm{CH} 2-\mathrm{N}\right) ; 1.79-$ $1.97(\mathrm{~m}, 6 \mathrm{H}, \mathrm{CH} 2-\mathrm{CH} 2-\mathrm{CH} 2-\mathrm{N}$; (O)C-CH2-CH2-CH2-N); 2.12 (s, $3 \mathrm{H}, \mathrm{CH} 3-(\mathrm{O}) \mathrm{C}) ; 2.72(\mathrm{t}, \mathrm{J}=6.0 \mathrm{~Hz}$, $2 \mathrm{H},(\mathrm{O}) \mathrm{C}-\mathrm{CH} 2-\mathrm{CH} 2-\mathrm{CH} 2-\mathrm{N})$; 3.47-3.63 (m, 8 H, CH2-CH2-CH2-N; (O)CCH2-CH2-CH2-N; CH3-CH2-N).

${ }^{13} \mathrm{C} \mathrm{NMR}(100 \mathrm{MHz}, \mathrm{CDCl} 3) \delta$ (ppm): $7.5(\mathrm{CH} 3-\mathrm{CH} 2-\mathrm{N}) ; 16.0 ; 19.8$ (CH2-CH2-CH2-N; (O)C-CH2-CH2-CH2$\mathrm{N})$; $20.8(\mathrm{CH} 2-\mathrm{CH} 2-\mathrm{CH} 2-\mathrm{N}) ; 30.1(\mathrm{C}(\mathrm{O})-\mathrm{CH} 3) ; 39.1$ ((O)C-CH2-CH2-CH2-N); 54.2; $56.3(\mathrm{CH} 2-\mathrm{CH} 2-\mathrm{CH} 2-\mathrm{N})$; $58.7(\mathrm{CH} 3-\mathrm{CH} 2-\mathrm{N}) ; 207.3(\mathrm{C}(\mathrm{O}))$.

\subsubsection{General procedure for halide ion metathesis by NTf2-anion (compound C)}

To a solution of halide salt $(68 \mathrm{mmol})$ in $20 \mathrm{~mL}$ of MilliQ water was added a solution of bis(trifluoromethane)sulfonimide lithium salt $(20 \mathrm{~g}, 69 \mathrm{mmol})$ in $20 \mathrm{~mL}$ of MilliQ water. The mixture was stirred for $2 \mathrm{~h}$ at room temperature, and the biphasic mixture was then separated. The organic phase was washed with water until the silver nitrate test was negative; the ionic liquid was then dried under vacuum at $80^{\circ} \mathrm{C}$ for $3 \mathrm{~h}$.

The TSIL was obtained as yellow viscous oil. 
2.2.4.1. C1a: [EPip-MIBK]NTf2:

N-ethyl-N-(4-methyl-2-oxopentyl)-piperidinium

bis(trifluoromethane)sulfonimide (yield 95\%).

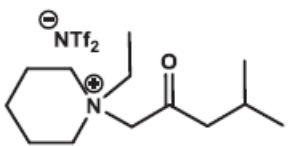

[EPip-MIBK] NTf $_{2}$

${ }^{1} \mathrm{H}$ NMR (400 MHz, CDCl3) $\delta$ (ppm): $0.90(\mathrm{~d}, 3 \mathrm{H}, \mathrm{J}=6.5 \mathrm{~Hz}, \mathrm{CH} 3) ; 1,23(\mathrm{t}, 3 \mathrm{H}, \mathrm{J}=7.5 \mathrm{~Hz}, \mathrm{CH} 3-\mathrm{CH} 2-\mathrm{N})$; $1.70(\mathrm{~m}, 2 \mathrm{H}, \mathrm{CH} 2) ; 1.87(\mathrm{~m}, 4 \mathrm{H}, \mathrm{CH} 2) ; 2.12(\mathrm{~m}, 1 \mathrm{H}, \mathrm{CH}) ; 2.39$ (d, $2 \mathrm{H}, \mathrm{J}=7.0 \mathrm{~Hz}, \mathrm{C}(\mathrm{O})-\mathrm{CH} 2) ; 3.44(\mathrm{~m}$, $2 \mathrm{H}, \mathrm{CH} 2-\mathrm{N}$ ); 3.62 (m, $2 \mathrm{H}, \mathrm{CH} 2-\mathrm{N}) ; 3.72(\mathrm{q}, 2 \mathrm{H}, \mathrm{J}=7.5 \mathrm{~Hz}, \mathrm{CH} 3-\mathrm{CH} 2-\mathrm{N}) ; 4.22$ (s, $2 \mathrm{H}, \mathrm{N}-\mathrm{CH} 2-\mathrm{C}(\mathrm{O})$ ). ${ }^{13} \mathrm{C}$ NMR $(100 \mathrm{MHz}, \mathrm{CDCl} 3) \delta$ (ppm): $7.4(\mathrm{CH} 3-\mathrm{CH} 2-\mathrm{N}) ; 19.5 ; 20.6(\mathrm{CH} 2) ; 22.1(\mathrm{CH} 3) ; 24.2(\mathrm{CH}) ; 49.5$ $(\mathrm{C}(\mathrm{O})-\mathrm{CH} 2) ; 55.0(\mathrm{CH} 3-\mathrm{CH} 2-\mathrm{N}) ; 60.0(\mathrm{CH} 2-\mathrm{N}) ; 61.8(\mathrm{~N}-\mathrm{CH} 2-\mathrm{C}(\mathrm{O})) ; 115.1-118.2-121.4-124.6$ (q, $\mathrm{J}=321 \mathrm{~Hz}, \mathrm{CF} 3) ; 201.6(\mathrm{C}(\mathrm{O}))$.

2.2.4.2. $\quad C 1 b: \quad[M I m-M I B K] N T f 2:$

N-methyl-N-(4-methyl-2-oxopentyl)imidazolium bis(trifluoromethane)sulfonimide (yield 95\%).

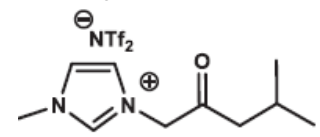

[MIm-MIBK]NTf

${ }^{1} \mathrm{H}$ NMR (400 MHz, CDCl3) $\delta(\mathrm{ppm}): 0.96(\mathrm{~d}, 3 \mathrm{H}, \mathrm{J}=6.5 \mathrm{~Hz}, \mathrm{CH} 3) ; 2.17(\mathrm{~m}, 1 \mathrm{H}, \mathrm{CH}) ; 2.51(\mathrm{~d}, 2 \mathrm{H}, \mathrm{J}=7.0$ $\mathrm{Hz}, \mathrm{C}(\mathrm{O})-\mathrm{CH} 2) ; 3.96(\mathrm{~s}, 3 \mathrm{H}, \mathrm{CH} 3-\mathrm{N}) ; 5.2(\mathrm{~s}, 2 \mathrm{H}, \mathrm{N}-\mathrm{CH} 2-\mathrm{C}(\mathrm{O})) ; 7.46$ (t, $1 \mathrm{H}, \mathrm{J}=2.0 \mathrm{~Hz}, \mathrm{CHAr}$ ); 7.57 (t, $1 \mathrm{H}, \mathrm{J}=2.0 \mathrm{~Hz}, \mathrm{CHAr}) ; 8.77(\mathrm{~s}, 1 \mathrm{H}, \mathrm{J}=2.0 \mathrm{~Hz}, \mathrm{CHAr})$.

${ }^{13} \mathrm{C}$ NMR (100 MHz, CD3OD) $\delta$ (ppm): 22.8 (CH3); 25.3 (CH); 36.6 (CH3-Ar); 49.1 (C(O)-CH2); 58.4 (N$\mathrm{CH} 2-\mathrm{C}(\mathrm{O})$ ); 116.4-119.6-122.8-126.0 (q, J = $321 \mathrm{~Hz}, \mathrm{CF} 3$ );124.4 (CHAr); 125.0 (CHAr); 139.0 (CHAr); $202.3(C(O))$.

2.2.4.3. C1c: [Py-MIBK]NTf2: N-(4-methyl-2-oxopentyl)pyridinium bis(trifluoromethane)sulfonimide (yield 95\%).

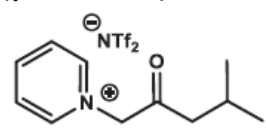

[Py-MIBK]NTf ${ }_{2}$

1H NMR (400 MHz, CDCl3) $\delta$ (ppm): $1.00(\mathrm{~d}, 3 \mathrm{H}, \mathrm{J}=6.5 \mathrm{~Hz}, \mathrm{CH} 3) ; 2.20(\mathrm{~m}, 1 \mathrm{H}, \mathrm{CH}) ; 2.61(\mathrm{~d}, 2 \mathrm{H}, \mathrm{J}=7.0$ $\mathrm{Hz}, \mathrm{C}(\mathrm{O})-\mathrm{CH} 2) ; 5.67(\mathrm{~s}, 2 \mathrm{H}, \mathrm{NCH} 2-\mathrm{C}(\mathrm{O})) ; 8.14(\mathrm{~m}, 2 \mathrm{H}, \mathrm{CHAr}) ; 8.66$ (tt, $1 \mathrm{H}, \mathrm{J}=8.0 \mathrm{~Hz}$; J= $1.5 \mathrm{~Hz}, \mathrm{CHAr}$ ); $8.74(\mathrm{~m}, 2 \mathrm{H}, \mathrm{CHAr})$.

${ }^{13} \mathrm{C} \mathrm{NMR}(100 \mathrm{MHz}, \mathrm{CD} 3 \mathrm{OD}) \delta$ (ppm): 22.9 (CH3); $25.3(\mathrm{CH}) ; 49.1$ (C(O)-CH2); 69.6 (N-CH2-C(O)); 116.4119.6-122.8-126.0 (q, J=321Hz, CF3);129.2 (CHAr); 147.3 (CHAr); 147.6 (CHAr); 201.4 (C(O)).

2.2.4.4. C2: [EPipAcP]NTf2: $N$-ethyl-N-acetophenone piperidinium bis(trifluoromethane)sulfonimide (yield 95\%).

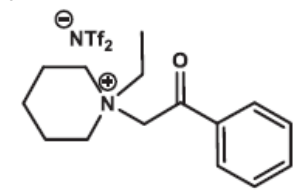

[EPip-AcPh]NTf

${ }^{1} \mathrm{H}$ NMR (400 MHz, CDCl3) $\delta(\mathrm{ppm}): 1.28(\mathrm{t}, 3 \mathrm{H}, \mathrm{J}=7.5 \mathrm{~Hz}, \mathrm{CH} 3-\mathrm{CH} 2-\mathrm{N}) ; 1.76(\mathrm{~m}, 2 \mathrm{H}, \mathrm{CH} 2) ; 1.94(\mathrm{~m}, 4$ $\mathrm{H}, \mathrm{CH} 2) ; 3.60(\mathrm{~m}, 2 \mathrm{H}, \mathrm{CH} 2-\mathrm{CH} 2-\mathrm{N}) ; 3.85(\mathrm{~m}, 4 \mathrm{H}, \mathrm{J}=7.5 \mathrm{~Hz}, \mathrm{CH} 3-\mathrm{CH} 2-\mathrm{N}, \mathrm{CH} 2-\mathrm{CH} 2-\mathrm{N})$; 4.85 (s, $2 \mathrm{H}, \mathrm{NCH} 2-$ $\mathrm{C}(\mathrm{O})) ; 7.52(\mathrm{t}, 2 \mathrm{H}, \mathrm{J}=7.5 \mathrm{~Hz}, \mathrm{CHAr}$ ); $7.67(\mathrm{t}, 1 \mathrm{H}, \mathrm{J}=7.5 \mathrm{~Hz}, \mathrm{CHAr}$ ); 8.00 (d, $2 \mathrm{H}, \mathrm{J}=7,5 \mathrm{~Hz}, \mathrm{CHAr}$ ).

${ }^{13} \mathrm{C} \mathrm{NMR}(100 \mathrm{MHz}, \mathrm{CDCl} 3) \delta$ (ppm): $7.7(\mathrm{CH} 3) ; 19.8 ; 20.8(\mathrm{CH} 2) ; 55.2(\mathrm{CH} 3-\mathrm{CH} 2-\mathrm{N}) ; 59.6(\mathrm{CH} 2-\mathrm{N}) ; 60.7$ (N-CH2-C(O)); 115.1-118.2-121.4-124.6 (q, J= 321 Hz, CF3); 128.3; 129.4 (CHAr); 133.7 (CAr); 135.5 (CHAr); 190.3 (C(O)). 
2.2.4.5. $\quad$ C3: $\quad[$ Epip-MAcPh]NTf2:

bis(trifluoromethane)sulfonimide (yield 95\%).

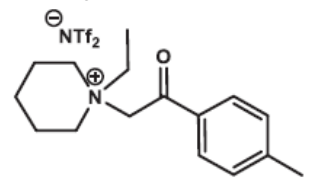

[EPip-MAcPh]NTf ${ }_{2}$

${ }^{1} \mathrm{H}$ NMR (400 MHz, CDCl3) $\delta$ (ppm): 1.28 (t, $\left.3 \mathrm{H}, \mathrm{J}=7.5 \mathrm{~Hz}, \mathrm{CH} 3-\mathrm{CH} 2-\mathrm{N}\right) ; 1.76(\mathrm{~m}, 2 \mathrm{H}, \mathrm{CH} 2) ; 1.94$ (m, 4 $\mathrm{H}, \mathrm{CH} 2) ; 3.60(\mathrm{~m}, 2 \mathrm{H}, \mathrm{CH} 2-\mathrm{CH} 2-\mathrm{N}) ; 3.85(\mathrm{~m}, 4 \mathrm{H}, \mathrm{J}=7.5 \mathrm{~Hz}, \mathrm{CH} 3-\mathrm{CH} 2-\mathrm{N}, \mathrm{CH} 2-\mathrm{CH} 2-\mathrm{N}) ; 4.85$ (s, $2 \mathrm{H}, \mathrm{NCH} 2-$ $\mathrm{C}(\mathrm{O})) ; 7.52(\mathrm{t}, 2 \mathrm{H}, \mathrm{J}=7.5 \mathrm{~Hz}, \mathrm{CHAr}$ ); $7.67(\mathrm{t}, 1 \mathrm{H}, \mathrm{J}=7.5 \mathrm{~Hz}, \mathrm{CHAr}$ ); 8.00 (d, $2 \mathrm{H}, \mathrm{J}=7.5 \mathrm{~Hz}, \mathrm{CHAr}$ ).

${ }^{13} \mathrm{C} \mathrm{NMR}(100 \mathrm{MHz}, \mathrm{CDCl} 3) \delta$ (ppm): 7.7 (CH3); 19.9; 20.8 (CH2); 21.8 (CH3-Ar); $22.0(\mathrm{CH} 3-\mathrm{CH} 2-\mathrm{N}) ; 51.2$ (CH3-CH2-N); 59.5 (CH2-N); 60.8 (N-CH2-C(O)); 115.1-118.2-121.4-124.6 (q, J = $321 \mathrm{~Hz}, \mathrm{CF} 3$ ); 128.5; 130.1 (CHAr); 131.2 (CAr); 147.1 (CAr); 189.7 (C(O)).

2.2.4.6. C4: [EPipPin]NTf2: N-ethyl-N-pinacolone piperidinium bis(trifluoromethane) sulfonimide (yield 95\%).

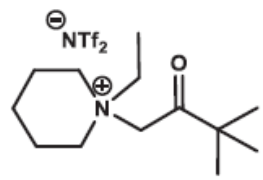

[EPip-Pin]NTf ${ }_{2}$

${ }^{1} \mathrm{H}$ NMR (400 MHz, CDCl3) $\delta$ (ppm): $1.23(\mathrm{~s}, 9 \mathrm{H}, \mathrm{CH} 3) ; 1.26$ (t, $\left.3 \mathrm{H}, \mathrm{J}=7.5 \mathrm{~Hz}, \mathrm{CH} 3-\mathrm{CH} 2-\mathrm{N}\right) ; 1.75(\mathrm{~m}, 2$ $\mathrm{H}, \mathrm{CH} 2) ; 1.88(\mathrm{~m}, 4 \mathrm{H}, \mathrm{CH} 2) ; 3.54(\mathrm{~m}, 2 \mathrm{H}, \mathrm{CH} 2-\mathrm{N}) ; 3.68(\mathrm{~m}, 2 \mathrm{H}, \mathrm{CH} 2-\mathrm{N}) ; 3.74(\mathrm{q}, 2 \mathrm{H}, \mathrm{J}=7.5 \mathrm{~Hz}, \mathrm{CH} 3-$ $\mathrm{CH} 2-\mathrm{N}) ; 4.40$ (s, $2 \mathrm{H}, \mathrm{N}-\mathrm{CH} 2-\mathrm{C}(\mathrm{O}))$.

${ }^{13} \mathrm{C} \mathrm{NMR}(100 \mathrm{MHz}, \mathrm{CDCl} 3) \delta(\mathrm{ppm}): 7.6(\mathrm{CH} 3-\mathrm{CH} 2-\mathrm{N}) ; 19.8 ; 20.8(\mathrm{CH}) ; 26.1$ (CH3); 45.1 (C(O)-C(CH3)3); $53.6(\mathrm{CH} 3-\mathrm{CH} 2-\mathrm{N}) ; 59.5(\mathrm{~N}-\mathrm{CH} 2-\mathrm{C}(\mathrm{O})) ; 60.1(\mathrm{CH} 2-\mathrm{N}) ; 115.1-118.2-121.5-124.7$ (q, J = $321 \mathrm{~Hz}$, CF3); 207.6 (C(O)).

\subsubsection{C5: [MIMPin]NTf2: N-pinacolone methylimidazolium bis(trifluoromethane)} sulfonimide (yield 95\%).

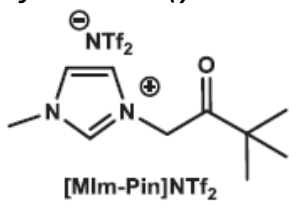

${ }^{1} \mathrm{H}$ NMR (400 MHz, CDCl3) $\delta$ (ppm): 1.25 (s, $\left.9 \mathrm{H}, \mathrm{CH} 3\right) ; 3.94$ (s, $\left.3 \mathrm{H}, \mathrm{CH} 3-\mathrm{Ar}\right) ; 5.31$ (s, $2 \mathrm{H}, \mathrm{N}-\mathrm{CH} 2-\mathrm{C}(\mathrm{O})$ ); $7.28(3,2 \mathrm{H}, \mathrm{CHAr}) ; 8.69(\mathrm{~s}, 1 \mathrm{H}, \mathrm{CHAr})$.

${ }^{13} \mathrm{C} \mathrm{NMR}(100 \mathrm{MHz}, \mathrm{CDCl} 3) \delta(\mathrm{ppm}): 26.0(\mathrm{CH} 3) ; 36.5(\mathrm{CH} 3-\mathrm{N}) ; 43.6((\mathrm{CH} 3) 3-\mathrm{C}-\mathrm{C}(\mathrm{O})) ; 53.9(\mathrm{C}(\mathrm{O})-\mathrm{CH} 2-\mathrm{N})$; 115.1-118.2-121.4-124.6 (q, J = 321 Hz, CF3); 122.8 (CHAr); 124.1 (CHAr); 137.7 (CHAr); 206.0 (C(O)).

2.2.4.8. C6: [EPip-P4K]NTf2: 1-ethyl-4-oxopentyl-piperidinium bis(trifluoromethane)

sulfonimide (yield 85\%).

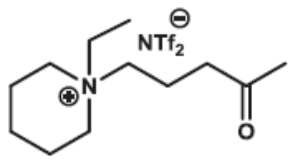

[EPip-P4K]NTf ${ }_{2}$

${ }^{1} \mathrm{H}$ NMR (400 MHz, CDCl3) $\delta$ (ppm): 1.34 (t, J =7.5 Hz, CH3-CH2-N); $1.71(\mathrm{~m}, 2 \mathrm{H}, \mathrm{CH} 2-\mathrm{CH} 2-\mathrm{CH} 2-\mathrm{N}) ; 1.80-$ $1.96(\mathrm{~m}, 6 \mathrm{H}, \mathrm{CH} 2-\mathrm{CH} 2-\mathrm{CH} 2-\mathrm{N}$; (O)C-CH2-CH2-CH2-N); 2.15 (s, $3 \mathrm{H}, \mathrm{CH} 3-(\mathrm{O}) \mathrm{C}) ; 2.64$ (t, J = $6.0 \mathrm{~Hz}$, $2 \mathrm{H},(\mathrm{O}) \mathrm{C}-\mathrm{CH} 2-\mathrm{CH} 2-\mathrm{CH} 2-\mathrm{N}) ; 3.21(\mathrm{~m}, 2 \mathrm{H},(\mathrm{O}) \mathrm{C}-\mathrm{CH} 2-\mathrm{CH} 2-\mathrm{CH} 2-\mathrm{N}) ; 3.25-3.37(\mathrm{~m}, 6 \mathrm{H}, \mathrm{CH} 2-\mathrm{CH} 2-\mathrm{CH} 2-\mathrm{N}$; $\mathrm{CH} 3-\mathrm{CH} 2-\mathrm{N})$. 
${ }^{13} \mathrm{C} \mathrm{NMR}(100 \mathrm{MHz}, \mathrm{CDCl}) \delta$ (ppm): $7.0(\mathrm{CH} 3-\mathrm{CH} 2-\mathrm{N}) ; 15.4 ; 19.5(\mathrm{CH} 2-\mathrm{CH} 2-\mathrm{CH} 2-\mathrm{N}$; (O)C-CH2-CH2-CH2$\mathrm{N}) ; 20.9(\mathrm{CH} 2-\mathrm{CH} 2-\mathrm{CH} 2-\mathrm{N}) ; 29.8(\mathrm{C}(\mathrm{O})-\mathrm{CH} 3) ; 38.6$ ((O)C-CH2-CH2-CH2-N); 54.7; $56.3(\mathrm{CH} 2-\mathrm{CH} 2-\mathrm{CH} 2-\mathrm{N})$; 59.2 (CH3-CH2-N); 115.1-118.3-121.5-124.7 (q, J= 321 Hz, CF3); 207.3 (C(O)).

\subsection{Extraction experiments}

$\mathrm{Ta}(\mathrm{V})$ stock solutions $(1-10 \mathrm{~g} / \mathrm{L})$ were prepared at the desired acidity $\left(\mathrm{H}_{2} \mathrm{SO}_{4} 0-9 \mathrm{M}\right)$ from $10000 \mathrm{mg} / \mathrm{L}$ ICP standard from SCP Science (aqueous solution of $\mathrm{NH}_{4} \mathrm{TaF}_{6}$ with $1 \%$ fluorhydric acid). A synthetic solution mimicking the leaching of capacitors was prepared from $\mathrm{Ta}(\mathrm{V})$ and of $\mathrm{Nb}, \mathrm{Fe}, \mathrm{Mn}, \mathrm{Ni}$ and $\mathrm{Ag}$ from $10000 \mathrm{mg} / \mathrm{L}$ and $1000 \mathrm{mg} / \mathrm{LICP}$ standards. The desired concentrations were prepared by dilution using ultrapure water (Milli-Q, Millipore, $18 \mathrm{M} \Omega / \mathrm{cm}$ ) and the acidity was adjusted with sulphuric acid. The initial concentrations of metals were measured by inductively coupled plasma/atomic emission spectroscopy (ICP/AES).

Extractions were performed by shaking defined volumes of TSIL and aqueous phases using a thermostated shaker (Infor-ht ${ }^{\circledast}$ ecotron) at $25^{\circ} \mathrm{C}$ and $400 \mathrm{rpm}$ for $1 \mathrm{~h}$. Aqueous to TSIL phase (Vaq/VIL) volumetric ratio of 1 or 2 has been used.

After the extraction, separation of the phases was assisted by centrifugation ( $4000 \mathrm{rpm}$ for $5 \mathrm{~min}$ ), and the aqueous phase was analysed by ICP/AES. The organic phase was submitted to a stripping step using ultrapure water (MilliQ, Millipore, $18 \mathrm{M} \Omega / \mathrm{cm}$ ). Back-extractions were performed at $25^{\circ} \mathrm{C}$ by contacting the loaded IL phase with water during $1 \mathrm{~h}$ (aqueous to IL phase volumetric ratio of 1 or 2). After separation by centrifugation ( $4000 \mathrm{rpm}$ for $10 \mathrm{~min}$ ), metals concentrations were measured in the aqueous phase by ICP/AES. All the experiments were carried out in duplicate measurements with a precision of $\pm 5 \%$. The efficiency of the TSIL to selectively extract Ta towards iron was evaluated from the following parameters. The distribution ratio (D) was determined using the following equation:

$$
D=\frac{[\mathrm{Ta}]_{\mathrm{IL}}}{[\mathrm{Ta}]_{\mathrm{aq}}}=\frac{\mathrm{C}_{\mathrm{i}}-\mathrm{C}_{\mathrm{f}}}{\mathrm{C}_{\mathrm{f}}} \times \frac{\mathrm{V}_{\mathrm{aq}}}{\mathrm{V}_{\mathrm{IL}}}
$$

$\mathrm{Ci}$ and $\mathrm{Cf}$ are the concentrations of the metal ions in the aqueous phase before and after extraction, respectively. Vaq is the volume of the aqueous phase and VIL is the volume of the ionic liquid phase. The extraction efficiency (\%E) was determined by using the following equation:

$$
\% \mathrm{E}=\frac{D}{D+\frac{\mathrm{V}_{\mathrm{aq}}}{\mathrm{V}_{\mathrm{IL}}}} \times 100 \%
$$

The stripping percentage (\%S) was calculated as follows for

$$
\% S=\frac{[\mathrm{Ta}]_{\mathrm{st}}}{[\mathrm{Ta}]_{\mathrm{IL}}} \times 100 \%
$$

[Ta]st and [Ta]IL are the concentrations of the metal ions in the stripping aqueous phase after stripping and the concentrations of the metal ions in the loaded TSILs phase before stripping, respectively. The stripped TSIL phases were reused in further cycles of the extraction-stripping process.

The separation factor $\left(\mathrm{SF}_{\mathrm{T}} / \mathrm{M}\right)$ is given by:

$$
S F=\frac{D_{\mathrm{Ta}}}{D_{\mathrm{M}}}
$$

$D_{T a}$ and $D_{M}$ are the distribution ratios of the Ta and $M$ the competitive ions, respectively. 


\section{Results and discussion}

\subsection{Synthesis}

The functionalized hydrophobic ionic liquids based on piperidium, imidazolium or pyridinium cations bearing ketone moieties have been synthesized according to the Scheme 1 or 2 .

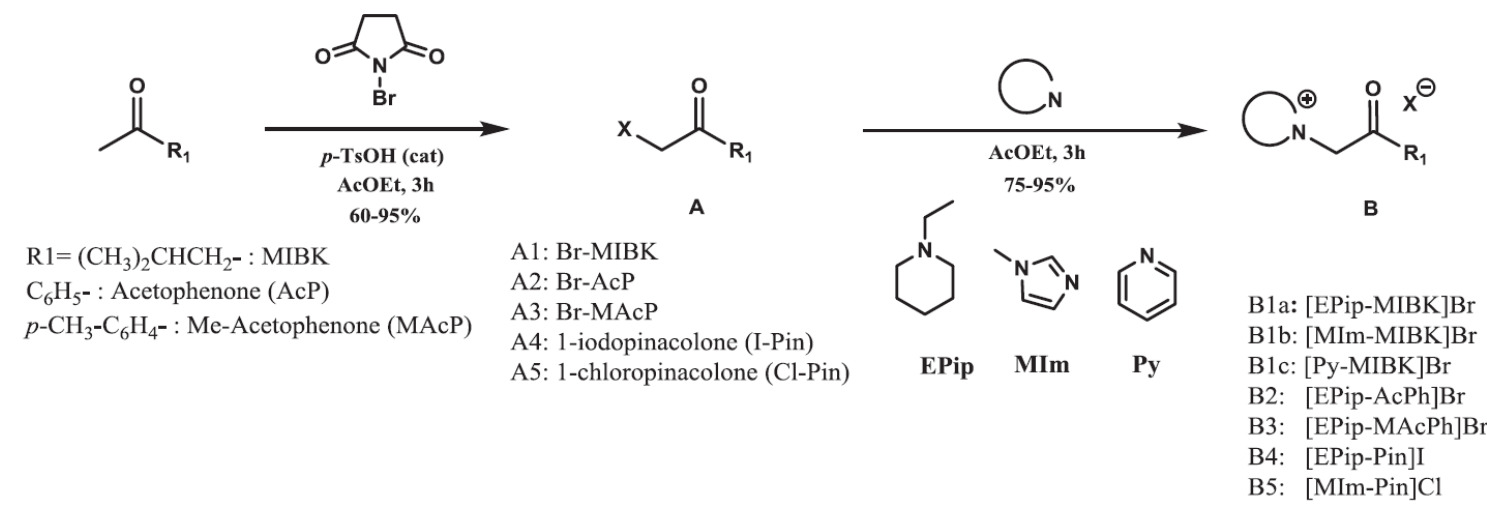

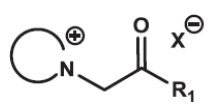

B

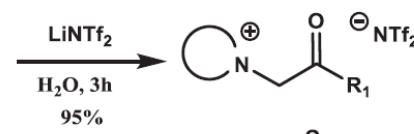

C1a: [EPip-MIBK]NTf

C1b: [MIm-MIBK]NTf ${ }_{2}$

C1c: [Py-MIBK]NTf ${ }_{2}$

C2: [EPip-AcPh]NTf ${ }_{2}$

C3: [EPip-MAcPh] $\mathrm{NTf}_{2}$

C4: [EPip-Pin] $\mathrm{NTf}_{2}$

C5: [MIm-Pin]NTf

Scheme 1. First synthetic strategy for bis(trifluoromethylsulfonyl)imide TSILs bearing a ketone functionality.

The first synthetic route (Scheme 1 ) is based on the use of $\alpha$-haloketone that is reacted with different amines. The precursors $A$ were prepared by reacting a ketone such as methyl iso-butyl ketone (MIBK), acetophenone (AcPh) or 4-methyacetophenone (MAcPh) with N-Bromosuccinimide and a catalytic amount of $p$-Toluenesulfonic acid. The synthesized $\alpha$-bromoketone or commercially available $\alpha$ haloketone were then reacted with heterocyclic amine such as ethylpiperidine (Epip), methylimidazolium (MIm) or pyridine (Py) to form the corresponding quaternary salts (compounds B). The metathesis with LiNTf $_{2}$ converted the halide anions into bis(trifluoro-methanesulfonyl)amide providing the desired hydrophobic ionic liquids (compounds $\mathrm{C}$ ).

In order to study chain length influence between the ketone and the cation of the IL a second synthetic route (Scheme 2) has been implemented.
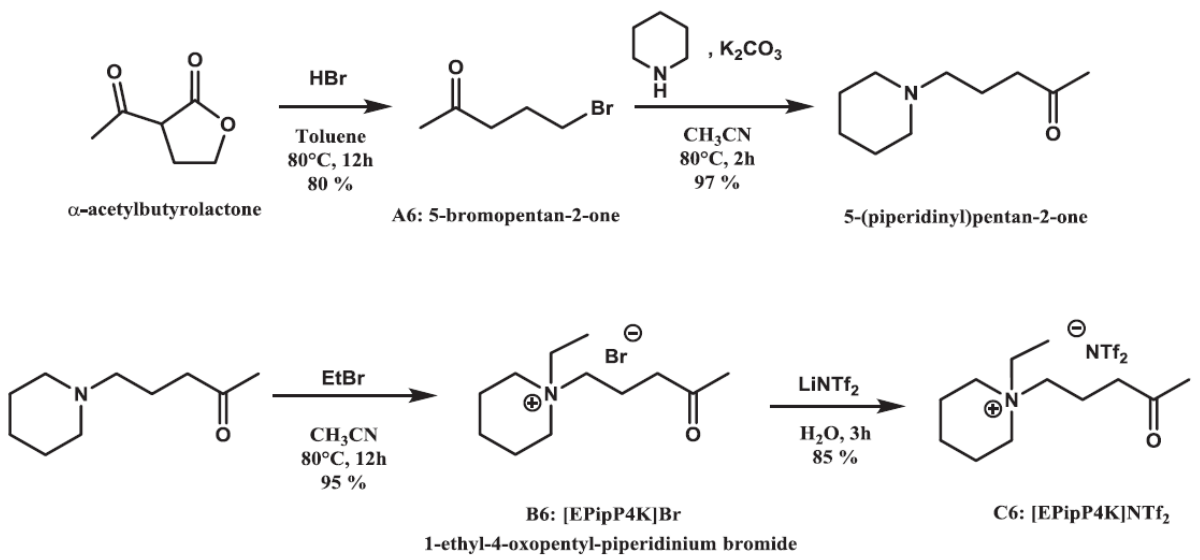

Scheme 2. Second synthetic strategy for bis(trifluoromethylsulfonyl)imide TSILs bearing a ketone functionality. 
Cleavage of acetylbutyrolactone with $\mathrm{HBr}$ provided 5-bromopentan-2-one that was then reacted with piperidine. Quaternization with ethylbromide was followed by metathesis as previously described, giving then access to the desired hydrophobic ionic liquid. The physicochemical properties of the TSILS bearing a ketone functionality are provided in the Table 1.

Table 1 Physicochemical properties of bis(trifluoromethylsulfonyl)imide (NTf2)-based TSILs ketone tested in this study.

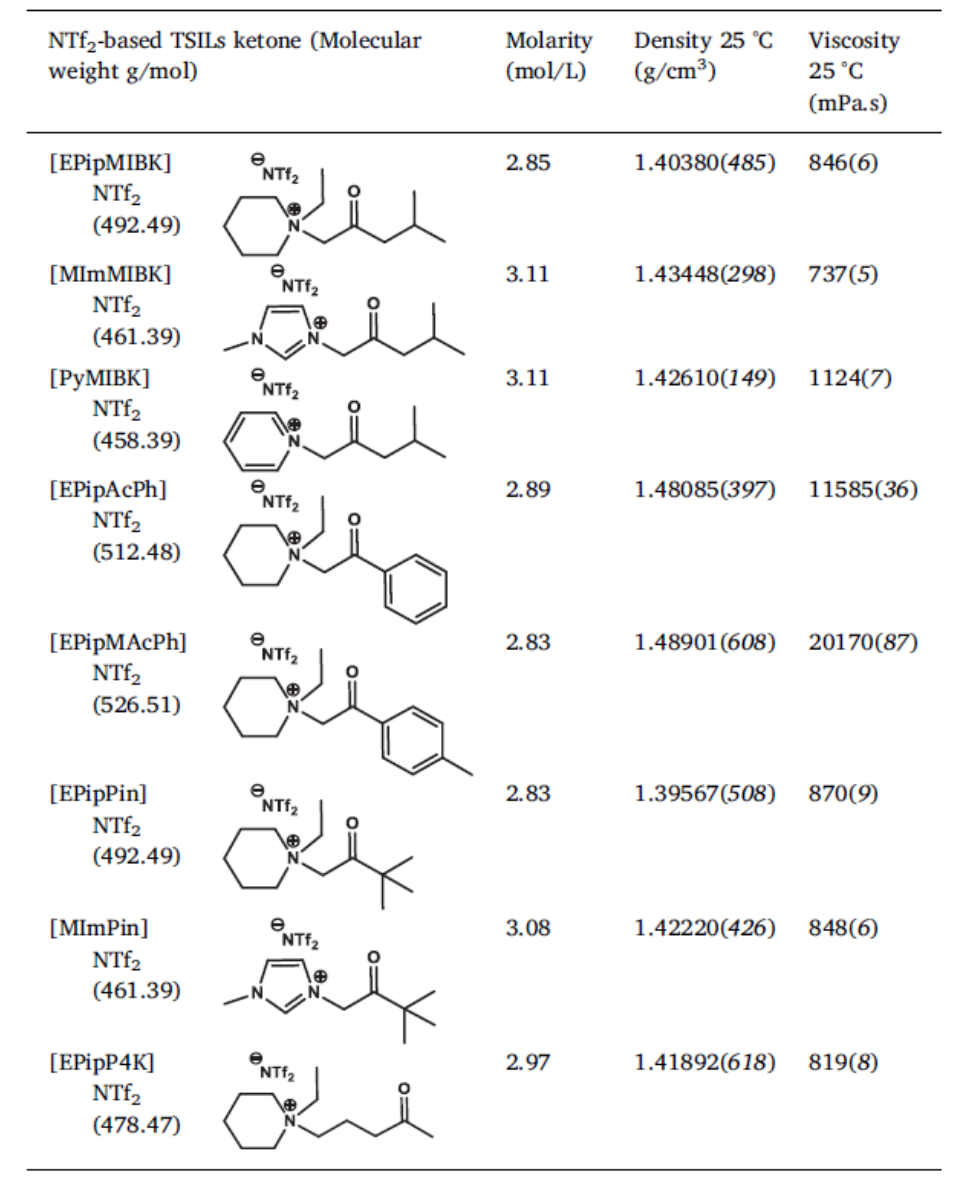

\subsection{Extraction experiments}

Liquid-liquid extraction is strongly influenced by hydrodynamic parameters such as viscosity. All TSILs synthesized are liquid at room temperature but they are viscous. Nevertheless, they present a sufficiently low viscosity to be directly employed as pure extracting phase/ extracting agents in liquidliquid extraction experiments. Aqueous to TSIL phase (Vaq/VIL) volumetric ratio of 1 has been used with a TSIL molar concentration of around $3 \mathrm{~mol} / \mathrm{L}$ for all the TSILs (Table 1), therefore it was assumed that the extraction capacities can be considered as comparable. The distribution ratios of Ta remain almost constant after $30 \mathrm{~min}$ at $25^{\circ} \mathrm{C}$ for the reference TSIL ([EPipMIBK]NTf2); indeed, it was assumed that it takes $30 \mathrm{~min}$ to reach the equilibrium state with this TSIL. In order to ensure the complete extraction and establish a comparison, all the studies have been carried out with a time of $1 \mathrm{~h}$.

The metal ion distribution ratios $\left(D_{T a}\right)$ and extraction efficiency $(\% \mathrm{E})$ for the all TSILs from a $6 \mathrm{M} \mathrm{H}_{2} \mathrm{SO}_{4}$ solution containing 1 or $7 \mathrm{~g} / \mathrm{L}$ of tantalum have been determined in order to establish the efficiency of the different TSILs. Effect of the ionic liquid cation and the ketone functionality have been investigated. 


\subsubsection{Effect of the ionic liquid cation and ketone functionality}

The first extraction experiments were conducted to investigate the effect of IL cation on the extraction behavior of $\mathrm{Ta}(\mathrm{V})$ from $6 \mathrm{M} \mathrm{H}_{2} \mathrm{SO}_{4}$ medium by the pure TSILs. Remarkable distribution ratios were obtained for the TSIL with a methyl isobutyl ketone. It was observed that the extraction efficiency is almost the same for piperidium, imidazolium or pyridinium cations bearing a similar ketone moiety (Table 2).

Table 2 Distribution ratios, extraction efficiency (\%E) and back extraction (\%S) for Ta. Influence of the IL cation.

\begin{tabular}{|c|c|c|c|}
\hline & $\mathrm{D}_{\mathrm{Ta}}$ & $\% \mathrm{E}$ & $\% \mathrm{~S}$ \\
\hline [EPipMIBK]NTf ${ }_{2}$ & 43.5 & $98 \%$ & 100 \\
\hline$[\mathrm{MImMIBK}] \mathrm{NTf}_{2}$ & 23.7 & $96 \%$ & 86 \\
\hline [РyMIBK]NTf ${ }_{2}$ & 20.9 & $95 \%$ & 86 \\
\hline
\end{tabular}

Extraction conditions: $[\mathrm{Ta}]=1 \mathrm{~g} / \mathrm{L},\left[\mathrm{H}_{2} \mathrm{SO}_{4}\right]=6 \mathrm{M}, \mathrm{Vaq} / \mathrm{VIL}=1$; Stripping conditions: Milli-Q water, Vaq/VIL=1

Ionic liquids functionalized by a ketone having an aromatic, isobutyl or tert-butyl group have been synthesized and evaluated in the context of $\mathrm{Ta}(\mathrm{V})$ from $6 \mathrm{M} \mathrm{H}_{2} \mathrm{SO}_{4}$ medium (Table 3).

Higher distribution ratio and extraction efficiency were obtained when using the ionic liquid with aliphatic ketone (isobutyl or tertbutyl).

Table 3 Distribution ratios, extraction efficiency (\%E) and back extraction (\%S) for Ta.Influence of the IL ketone functionality.

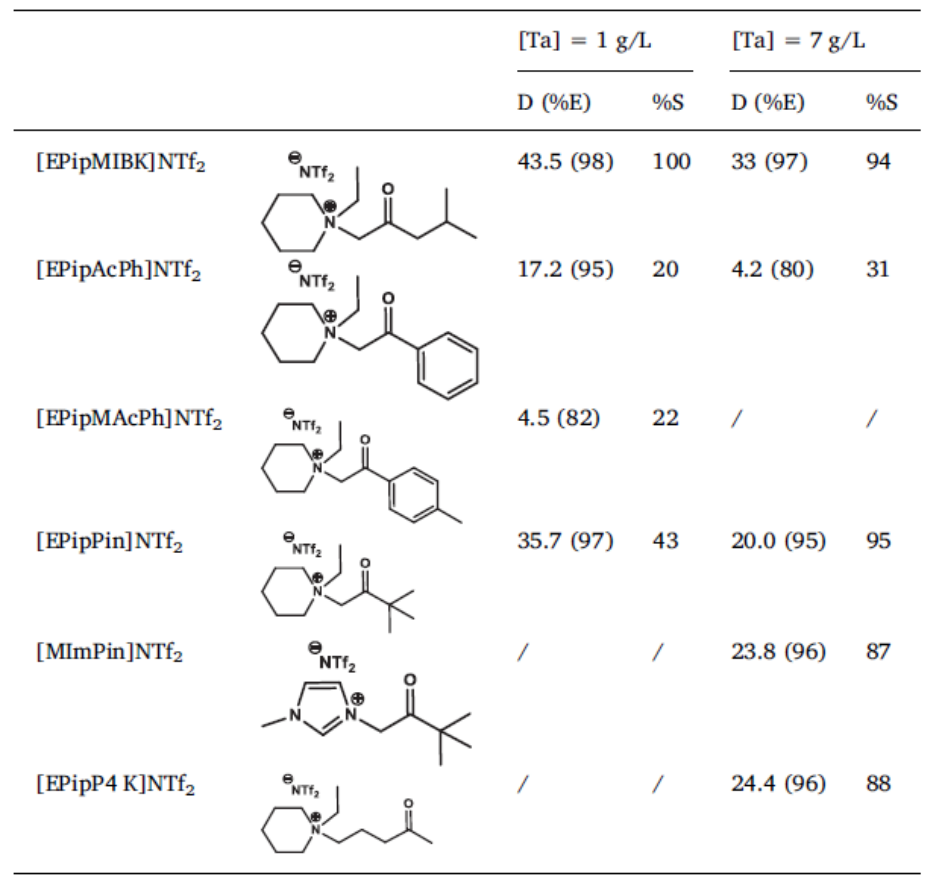

Extraction conditions: $[\mathrm{Ta}]=1$ or $7 \mathrm{~g} / \mathrm{L},\left[\mathrm{H}_{2} \mathrm{SO}_{4}\right]=6 \mathrm{M}, \mathrm{Vaq} / \mathrm{VIL}=1$; Stripping conditions: Milli-Q water, Vaq/VIL=1. 
The ionic liquid having the MIBK group remains slightly better than the ionic liquid having a tert-butyl group. When the ketones are aromatic, the extraction falls, this can be due to the higher viscosity of these ionic liquids bearing aromatic ketone; this has an impact on the hydrodynamic parameters of the extraction. A study on the phase transfer kinetics indicated that equilibrium was attained quickly for the EPipMIBK (Figure SI-1). The amount of Ta extracted increased rapidly, the values obtained after 30 min did not change significantly, a plateau is observed with an extraction efficiency of approximately $98 \%$. In comparison, for the aromatic ketone [EPipAcPh]NTf2 the equilibrium is not reached after 60 min and the difference in viscosity may explain this slow mass transfer.

When the results are compared for EPipMIBK, EPipPin and EPipP4 K it appears that the spacer between the IL cation and the ketone functionality do not influence the extraction performances.

For a similar aqueous/IL volumetric ratio of 1 , the extraction efficiency decreases slightly when the tantalum concentration increases from 1 to $7 \mathrm{~g} / \mathrm{L}$. However, despite this diminution more than $97 \%$ of the initial amount of Ta can be extracted. From the different ILs bearing ketone functionality the mimic of the methyl isobutyl ketone has been suggested to be most effective.

\subsubsection{Stripping and reusability studies}

After quantitative extraction of Ta into the ionic liquid phase, the stripping was carried out using Milli$Q$ water. Complete back extraction of Ta into the aqueous phase was possible within one or two single step with aqueous/IL volumetric ratio of 1 . The same stripping behavior has been observed for the various ionic liquids except for the ionic liquids bearing aromatic ketones; once again, this is related to their higher viscosity. After complete stripping of the metal ion, the ionic liquid phase is reused for further extraction step. It was observed (Fig. 1) that the extraction efficiency remains almost the same using the recycled ionic liquid (97\%, 96\% and $97 \%$ for recycle number 1, 2 and 3, respectively).

Consequently, the ionic liquid can be re-used at least three times without any change in extraction efficiency. The extraction efficiency of the ionic liquid phase remains intact after recycling.

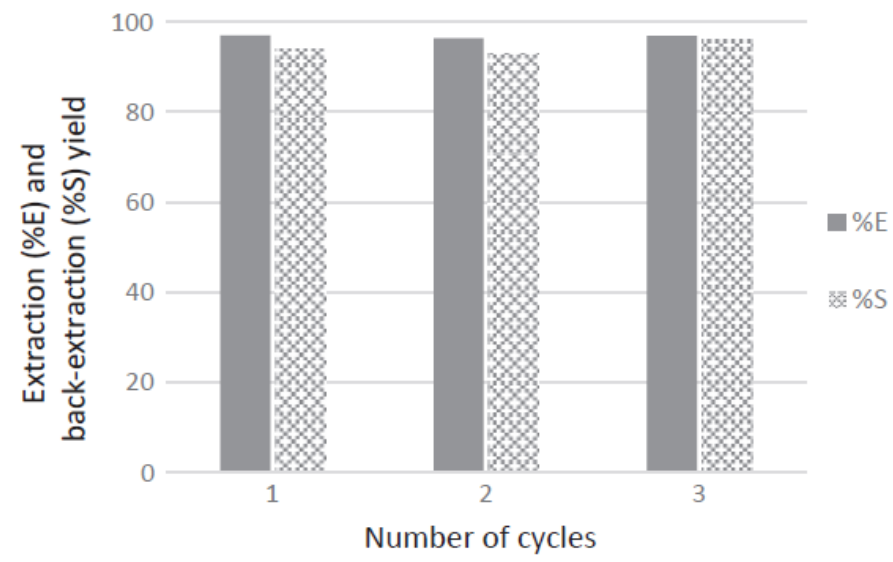

Fig. 1. $\mathrm{Ta}(\mathrm{V})$ extraction and back-extraction percentages for [EPipMIBK][NTf2] Organic phase: pure [EPipMIBK][NTf2]. Aqueous phase: $[\mathrm{Ta}]=1 \mathrm{~g} / \mathrm{L}\left[\mathrm{H}_{2} \mathrm{SO}_{4}\right]=6 \mathrm{M}$, Vaq/ VIL $=1$. Stripping: Milli-Q water, Vaq/VIL=1.

\subsubsection{Extraction of Ta from multi-element solutions}

The extraction studies for the [EPipMIBK][NTf2] were extended to competitive ions and as function of the sulfuric acid concentration in order to explore the use of the TSIL for the separation of Ta from solutions mimicking acidic leach liquors from ores or Waste of Electrical and Electronic Equipments (WEEE).

The variation of the distribution ratio of pure TSIL and MIBK as a function of sulfuric acid concentration is shown in Fig. 2. D values vary from 0.1 to 43 as the concentration of sulfuric acid is varied from 0 to 
$9 \mathrm{M}$. As described in the literature, tantalum is not extracted at low acidity $[5,22]$. Its extraction starts only with a concentration of $3 \mathrm{M}$ of sulfuric acid (91\%).

The extraction of tantalum is higher than $91 \%$ for a $\mathrm{H}_{2} \mathrm{SO}_{4}$ concentration of about $3 \mathrm{M}$, with almost no niobium extracted. The efficiencies of extractions of tantalum increase up to $97 \%$ when the $\mathrm{H}_{2} \mathrm{SO}_{4}$ is higher than $4 \mathrm{M}$. Interestingly, the efficiency of extractions of niobium increase only slightly with a maximum of about $15 \%$. This difference in their extraction performance could be used for the separation of the two metals. Indeed, the separation factor $\mathrm{SF}_{\mathrm{Ta}} / \mathrm{Nb}$ is higher than 100 between 3 and $9 \mathrm{M} \mathrm{of}_{2} \mathrm{SO}_{4}$. It is interesting to compare these results with those observed when using the traditional MIBK. Fig. 2 shows that extraction trend is similar to that of the MIBK extractant.

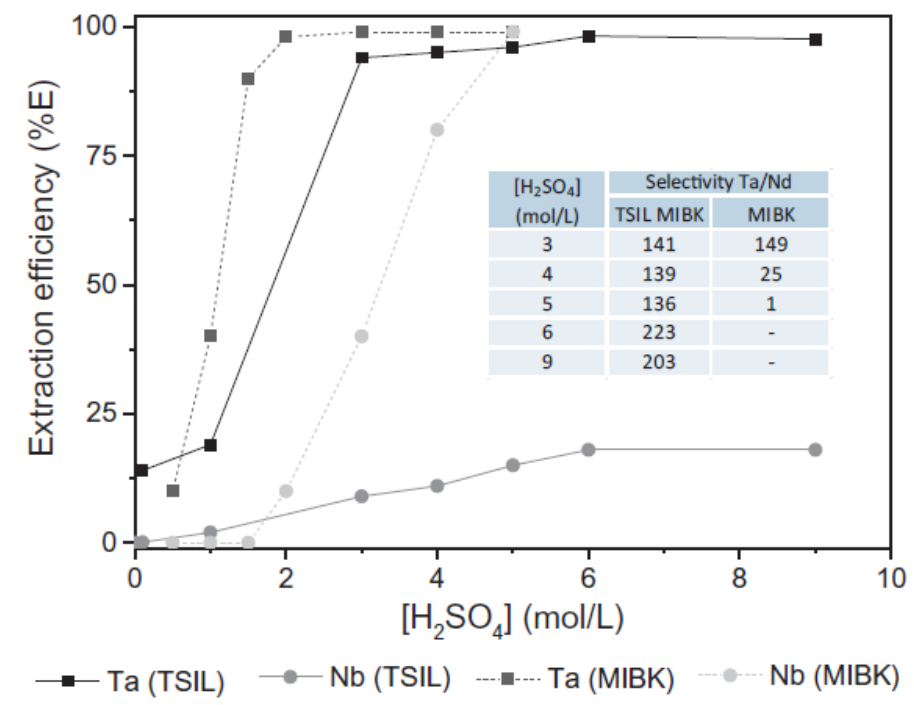

Fig. 2. Variation of the extraction efficiency of $\mathrm{Ta}$ and $\mathrm{Nb}$ and separation factor $\mathrm{SF}_{\mathrm{Ta}} / \mathrm{Nb}$ as a function of sulfuric acid concentration. Organic phase: pure MIBK or pure [EPipMIBK] [NTf2]. Aqueous phase: $[\mathrm{Ta}]=1 \mathrm{~g} / \mathrm{L}$ and $[\mathrm{Nb}]=1 \mathrm{~g} / \mathrm{L},[\mathrm{H} 2 \mathrm{SO}$ ] $=$ $0-9 \mathrm{M}, \mathrm{Vaq} / \mathrm{VIL}=1$.

When increasing the concentration of sulfuric acid, an increase of $D$ values is also observed for the conventional MIBK extractant. In comparison to MIBK, the TSIL is able to extract Ta for higher sulfuric acid concentrations. In addition, a better Ta/Nd selectivity is observed for the TSIL. The TSIL has demonstrated to be very a versatile compound for the extraction of Ta, yielding high $\mathrm{D}$ values and extraction efficiencies even when the $\mathrm{H} 2 \mathrm{SO} 4$ was higher than $5 \mathrm{M}$. With the traditional MIBK, the extraction at high concentration ( $>5 \mathrm{M}$ ) is not possible due its degradation. An important point is that for all the extractions experiments, complete back extraction of Ta from the TSIL into the aqueous phase was possible within one or two stages with Milli- $Q$ water.

To deepen the potential of this TSIL, the extraction of the Ta with respect to $\mathrm{Nb}, \mathrm{Fe}, \mathrm{Ni}, \mathrm{Mn}$ and $\mathrm{Ag}$, which can also be found as competitive ions in acidic leach liquors of capacitors, has been investigated. As illustrated in the Fig. 3, the competitive ions such as $\mathrm{Nb}, \mathrm{Fe}, \mathrm{Ni}, \mathrm{Mn}$ and $\mathrm{Ag}$, are left in the raffinate when tantalum is extracted with the TSIL MIBK.

Regardless of the concentration of sulfuric acid, the TSIL remains selective for tantalum with values of separation factor $\mathrm{SF}_{\mathrm{T}} / \mathrm{M}$ ranging from 30 to 1400 , depending on the acidity and the metallic cations considered.

After complete stripping of the metal ion, the ionic liquid phase is reused for further extraction step. The extraction pattern and distribution ratios are similar to those obtained before the recycling of the TSIL (see Figure SI-2 supporting information).

\subsubsection{Extraction mechanism}

The extraction of niobium and tantalum with MIBK was related to a salting out effect. The extend of extraction was found to increase with increase in the concentration of $\mathrm{H}_{2} \mathrm{SO}_{4}$. [23] The species that is responsible for extraction might constitute an ion association complex. 


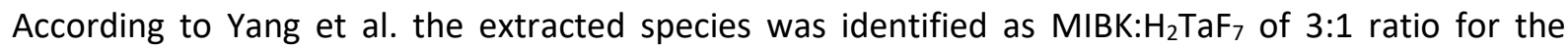
extraction of tantalum from sulphuric media containing hydrofluoric acid.[24] MIBK dissolves the tantalum and niobium compounds into the ketone while leaving impurities in the aqueous solution. Both niobium and tantalum are extracted at high concentration of $\mathrm{H}_{2} \mathrm{SO}_{4}$, but only tantalum is extracted at lower acidity.[22,25] Niobium complexes in the solution are stronger Lewis acids than those of tantalum. Therefore, the extraction of niobium with MIBK requires stronger solution acidity than that of tantalum which makes it possible to envisage their separation and also in regards to other competitive ions. $[26,27]$

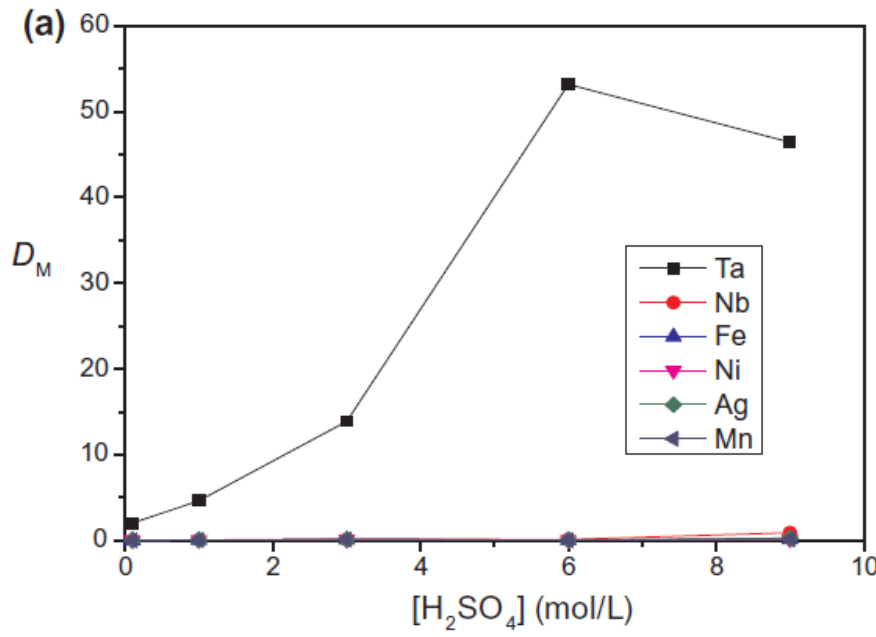

(b)

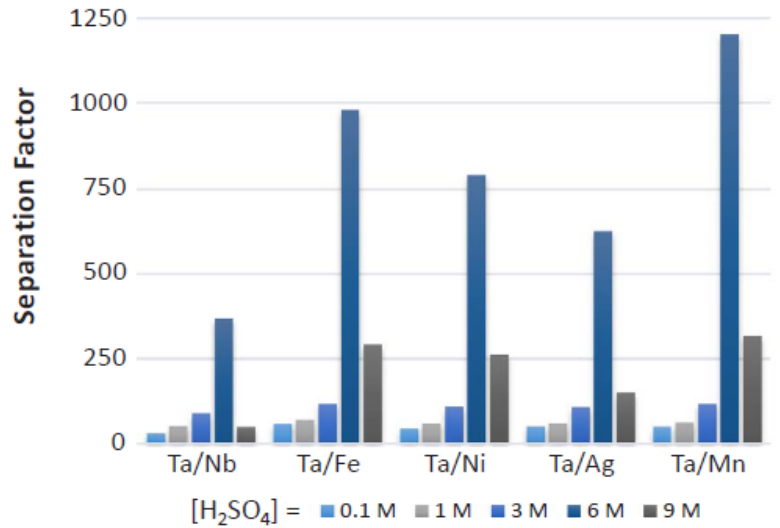

Fig. 3. a) Variation of the distribution ratio of $\mathrm{Ta}, \mathrm{Nb}, \mathrm{Fe}, \mathrm{Ni}, \mathrm{Ag}$ and $\mathrm{Mn}$ as a function of sulfuric acid concentration; b) Separation factor $\left(\mathrm{SF}_{\mathrm{Ta}} / \mathrm{M}\right)$ as a function of sulfuric acid concentration. Organic phase: pure [EPipMIBK] [NTf2]. Aqueous phase: [metals] $=2.5 \mathrm{~g} / \mathrm{L},\left[\mathrm{H}_{2} \mathrm{SO}_{4}\right]=0-9 \mathrm{M}, \mathrm{Vaq} / \mathrm{VIL}=1$.

As shown in the Fig. 2 comparable extraction behaviour to those with MIBK is observed for the [EPipMIBK][NTf2] allowing to consider a related mechanism.

In order to better understand the extraction mechanism involved in the TSIL media, the possibility of cation or anion exchange in the aqueous phase have been studied. NMR analysis of the TSIL and aqueous phases was studied following the ${ }^{19} \mathrm{~F}$ nucleus for the NTf2 anion and ${ }^{1} \mathrm{H},{ }^{13} \mathrm{C}$ for the [EPipMIBK] cation. The NMR analysis of the aqueous phase indicate the presence of both anion and cation as trace. The amount of [EPipMIBK][NTf2] TSIL transferred in the aqueous phase was estimated by TOC analysis with a value of around $5100 \mathrm{ppm}$ of organic in the aqueous phase which is corresponding to less than $0.4 \%$ of the initial amount of the TSIL (Table 4). This loss did not affect the extraction efficiency as can be seen with Fig. 1 and the reusability studies. An equivalent transferred amount of TSIL into the aqueous phase has been found when the initial concentration of Tantalum was 1 or $7 \mathrm{~g} / \mathrm{L}$ and also without any tantalum suggesting that this value is correlated to the solubilisation of the TSIL in the aqueous phase more than a loss due to a cation or anion exchange (Table SI-1 supporting information). 
The aqueous and TSIL phases remained well separated, forming biphasic systems with any variation of volume phases after the extraction process. The [EPipMIBK] [NTf2] used for liquid-liquid extraction is not miscible with water, but is hygroscopic therefore the solubility of water in TSIL can be quite high. The water concentration in the TSIL phase before and after extraction was measured by Karl Fisher (Table 4). The high solubility of water in the TSIL could allow to overcome problems related to dehydration of the metal cations one of the limiting factors during extraction. However, it would be needed to better understand the role of water in the IL phase, and its possible interactions with the aggregation properties as has recently been demonstrated in conventional organic extractant/diluent systems [28].

Table 4 Characterization in the TSIL and aqueous phases before and after extraction.

\begin{tabular}{|c|c|c|c|}
\hline & & Before extraction & After extraction \\
\hline \multirow[t]{2}{*}{ TSIL phase } & Acidity $\mathrm{H}^{+}(\mathrm{mol} / \mathrm{L})$ & - & $0.104(26)$ \\
\hline & $\mathrm{H}_{2} \mathrm{O}$ content (ppm) & $4412(102)$ & $18995(488)$ \\
\hline \multirow[t]{2}{*}{ Aqueous phase } & Acidity $\mathrm{H}^{+}(\mathrm{mol} / \mathrm{L})$ & $12.36(38)$ & $12.30(25)$ \\
\hline & TOC (ppm) & - & $5112(64)$ \\
\hline
\end{tabular}

Extraction conditions: Organic phase: pure [EPipMIBK][NTf2]. Aqueous phase: $[\mathrm{Ta}]=1 \mathrm{~g} / \mathrm{L},\left[\mathrm{H}_{2} \mathrm{SO}_{4}\right]=6 \mathrm{M}, \mathrm{Vaq} / \mathrm{VIL}=1$.

After contacting the acidic aqueous phase and the TSIL phase, a part of the acid is extracted into the TSIL phase (Table 4). Proton transfer from the aqueous phase to the IL phase represents less than $1 \%$ of the initial acid concentration. As for the amount of water extracted, the understanding of the role of extracted acid during the extraction step should be further studied [29].

In regard to the different statements and behaviours observed by studying the TSIL mechanism exchange it appears that the anion or cation exchange mechanisms are probably not the driving force of the extraction. A possible mechanism for extraction of Tantalum ion from the aqueous acidic phase to the ionic liquid phase is the neutral / solvation mechanism. Several other investigations such as EXAFS or computational procedures could help to identify the role of water and acid in this process [29-31].

\section{Conclusion}

The objective of this study was to investigate the potential of hydrophobic TSILs bearing ketone in biphasic systems for the extraction of tantalum from sulfuric acid medium. In that context, we have reported here an efficient way to synthesize a novel family of TSILs bearing various ketone groups and cations. The extraction behavior of Ta with these TSILs was investigated for comparison and the mimic of the methyl isobutyl ketone was suggested to be most effective. The results described here show that high $D$ values can be achieved for the selective separation of Ta. Such system can be used as an alternative to the methyl-iso-butyl ketone which is the most commonly used extractant for the separation and purification of tantalum by solvent extraction.

The mechanism extraction pathway is probably not due to both the anion and cation exchange mechanism, as suggested by the analyses after the extraction of an aqueous acid solution with and without tantalum.

Comparable extraction behaviour to those with MIBK has been observed for the [EPipMIBK][NTf2] allowing to consider a related mechanism.

The recovery process for Ta after extraction was performed with water and recyclability was successfully established.

Furthermore, another interesting aspect is that the ketone functionality of the [EPipMIBK][NTf2] is stable over a wide electrochemical potential window and no reduction of the ketone functionality is observed as shown on voltammogram on a on vitrous carbon in the TSILs (Figure SI-3 see supporting information). This particularity also makes it possible to envisage the recovery of the Ta by electrodeposition. The feasibility for the electrodeposition of Ta using such system will be reported in due course. 


\section{Acknowledgements}

The authors thank the Labex Project CheMISyst (ANR-10-LABX-05- 01) and the Agence Nationale de la Recherche through the SILEXE project (ANR-13- CDII- 0010) for financial support.

\section{Appendix A. Supplementary material}

Supplementary data associated with this article can be found, in the online version.

\section{References}

[1] P. Černý, T.S. Ercit, in: P. Möller, P. Černý, F. Saupé, (Eds.), Lanthanides, Tantalum and Niobium, 1989, pp. 27-79.

[2] Albrecht, W. W. In Lanthanides, Tantalum and Niobium; Möller, P.; Černý, P.; Saupé, F. Eds., 1989; pp. 345-358.

[3] D.A.R. Mackay, G.J. Simandl, Mineral. Deposita 49 (2014) 1025-1047.

[4] H. Zhou, S. Zheng, Y. Zhang, Hydrometallurgy 80 (2005) 83-89.

[5] Z. Zhu, C.Y. Cheng, Hydrometallurgy 107 (2011) 1-12.

[6] J.G. Huddleston, H.D. Willauer, R.P. Swatloski, A.E. Visser, R.D. Rogers, Chem. Commun. (1998) $1765-1766$.

[7] J.F. Brennecke, E.J. Maginn, Aiche J. 47 (2001) 2384-2389.

[8] A.E. Visser, W.M. Reichert, R.P. Swatloski, H.D. Willauer, J.G. Huddleston, R.D. Rogers, lonic Liquids: Industrial Applications for Green Chemistry vol. 818, American Chemical Society, Washington, DC, 2002.

[9] S.H. Dai, Y.E. Ju, C. Barnes, J. Chem. Soc. Dalton Trans. (1999) 1201-1202.

[10] X.Q. Sun, H.M. Luo, S. Dai, Talanta 90 (2012) 132-137.

[11] X.Q. Sun, H.M. Luo, S. Dai, Chem. Rev. 112 (2012) 2100-2128.

[12] I. Billard, A. Ouadi, C. Gaillard, Anal. Bioanal. Chem. 400 (2011) 1555-1566.

[13] A. Rout, J. Kotlarska, W. Dehaen, K. Binnemans, Phys. Chem. Chemical Phys. 15

(2013) 16533-16541.

[14] T. Sukhbaatar, S. Dourdain, R. Turgis, J. Rey, G. Arrachart, S. Pellet-Rostaing, Chemical Commun. 51 (2015) 15960-15963.

[15] M.L. Dietz, Separat. Sci. Technol. 41 (2006) 2047-2063.

[16] S. Panja, P.K. Mohapatra, S.C. Tripathi, P.M. Gandhi, P. Janardan, Separat. Purifi. Technol. 96 (2012) 289-295.

[17] R. Turgis, G. Arrachart, V. Dubois, S. Dourdain, D. Virieux, S. Michel, S. Legeai, M. Lejeune, M. Draye, S. Pellet-Rostaing, Dalton Trans. 45 (2016) 1259-1268.

[18] I. Billard, A. Ouadi, C. Gaillard, Dalton Trans. 42 (2013) 6203-6212.

[19] C.H.C. Janssen, N.A. Macias-Ruvalcaba, M. Aguilar-Martinez, M.N. Kobrak, Int. Rev. Phys. Chem. 34 (2015) 591-622.

[20] S.Z. El Abedin, H.K. Farag, E.M. Moustafa, U. Welz-Biermanny, F. Endres, Phys. Chem. Chemical Phys. 7 (2005) 2333-2339.

[21] M. Nahra, L. Svecova, E. Chainet, Electrochim. Acta 182 (2015) 891-899.

[22] T. Okada, Manufacturing of special niobium oxides for optical and ceramic applications, in: International Symposium on Niobium 2001, 2001, Orlando, Florida, USA.

[23] C.K. Gupta, A.K. Suri, Extractive Metallurgy of Niobium, CRC Press, Boca Raton, Florida, USA, 1993.

[24] X. Yang, X. Wang, C. Wei, S. Zheng, Y. Zhang, Solvent Extract. Res. Develop. Japan 20 (2013) 159168.

[25] S.N. Bhattacharyya, B. Ganguly, Solvent Extract. Ion Exchange 2 (1984) 699-740.

[26] A. Agulyansky, Chemistry of Tantalum and Niobium Fluoride Compounds, Elsevier Amsterdam, The Netherlands, 2004.

[27] X. Yang, X. Wang, C. Wei, S. Zheng, Q. Sun, D. Wang, Hydrometallurgy 131 (2013) 34-39. 
[28] J. Rey, S. Dourdain, J.F. Dufrêche, L. Berthon, J.M. Muller, S. Pellet-Rostaing, T. Zemb, Langmuir 32 (2016) 13095-13105.

[29] C. Gaillard, V. Mazan, S. Georg, O. Klimchuk, M. Sypula, I. Billard, R. Schurhammer, G. Wipff, Phys. Chem. Chemical Phys. 14 (2012) 5187-5199.

[30] A. Maiti, A. Kumar, R.D. Rogers, Phys. Chem. Chemical Phys. 14 (2012) 5139-5146.

[31] M.G. Freire, L.M.N.B.F. Santos, I.M. Marrucho, J.A.P. Coutinho, Molten Salts and lonic Liquids: Never the Twain? John Wiley \& Sons, United States of America, 2009. 